\title{
Direct Photon-Hadron Correlations Measured with PHENIX
}

\author{
Megan Connors for the PHENIX Collaboration \\ Department of Physics and Astronomy, Stony Brook University, Stony Brook NY, 11794
}

\begin{abstract}
Direct photon-hadron correlations greatly improve our ability to perform jet tomography in heavy-ion collisions because the momentum of the direct photon can be used to constrain the initial momentum of the opposing jet. By comparing the spectrum of away-side hadrons observed in heavy ion collisions to the spectrum seen in nucleon collisions we can quantify the medium modification to the fragmentation function due to energy loss of the away-side parton.

High $p_{T}$ direct photon-hadron correlations have been measured with the PHENIX detector using a statistical subtraction method to remove the photon contribution from meson decays. The increased integrated luminosity in the most recent Au+Au RHIC run at $\sqrt{s_{N N}}=200 \mathrm{GeV}$ provides substantially improved statistical precision and enhances the kinematic reach. These measurements are compared to PHENIX $p+p$ results and several theoretical models of energy loss. In addition, we compare direct photon-hadron and $\pi^{0}$-hadron correlations.
\end{abstract}

Direct photon - jet correlations are considered a "golden channel" for studying jet tomography since the transverse momentum of the jet is approximately balanced by the transverse momentum of the photon and photons do not interact with the medium created at RHIC. The lack of medium modification to the direct photons has previously been shown by the PHENIX direct photon $R_{A A}$ measurement [1], which shows that $R_{A A}$ of direct photons is consistent with 1 out to at least $14 \mathrm{GeV} / \mathrm{c}$ in $p_{T}$. Direct photon - hadron correlations also allow measurement of the fragmentation function of the opposing parton [2]. The fragmentation function is $D_{q}(z)=d N(z) /\left(N_{\text {evt }} d z\right)$, where $z=p_{\text {hadron }} / p_{\text {jet }}$. For $\gamma_{\text {direct }}-h$ correlations, $z$ is approximately known, since the transverse momentum of the photon has the same magnitude as the opposing jet momentum to leading order. At NLO, however, the $k_{T}$ effect is important [3].

The measurement of these correlations, however, is complicated by the need to separate correlations involving direct photons from those involving inclusive photons. In heavy ion collisions, one of the largest sources of photons is decay photons, mostly from $\pi^{0} \rightarrow \gamma \gamma$. Direct photons are defined as all photons which do not result from a hadronic decay process since the decay photons are the only contribution subtracted from the inclusive sample. Prompt photons are the direct result of a hard scattering such as Compton scattering $(q g \rightarrow q \gamma)$ or quark-antiquark annihilation. Since Compton scattering is dominant, the away-side fragmentation is mostly quark jet fragmentation. Photons from jet fragmentation and medium-induced photons also contribute to the inclusive sample. To measure $\gamma_{\text {direct }}-h$ correlations, the contribution of decay photons are subtracted from inclusive $\gamma-h$ correlations via a statistical subtraction method. More details on the method and results from the 2004 data have been presented elsewhere [4]. First, inclusive $\gamma-h$ correlations are determined by measuring the angle between photons detected in the electromagnetic calorimeter and charged hadrons measured in the PHENIX tracking system. The 
measured inclusive photon yield can be written as:

$$
Y_{\mathrm{inc}}=\frac{N_{\mathrm{dir}} Y_{\mathrm{dir}}+N_{\mathrm{dec}} Y_{\mathrm{dec}}}{N_{\mathrm{inc}}} .
$$

The conditional yield here is defined as $Y=N_{\text {pair }} / N_{\text {trig }}$. To determine the decay contribution, first, $\pi^{0}-h$ correlations are measured. To translate $\pi^{0}-h$ correlations to $\gamma_{\text {decay }}-h$, a Monte Carlo simulation is used to determine the probability that a pion decays into a photon in a particular $p_{T}$ bin. A correction is applied in $A u+A u$ to account for the contribution from the $\eta$ decays based on the $p+p$ measurement. Higher mass decays are accounted for in the systematic error based on PYTHIA. Once the yield from $\gamma_{\text {decay }}-h$ is determined, the subtraction described in Equation 2. which is Equation 1 rearranged, is performed, where $R_{\gamma}=N_{\text {inc }} / N_{\text {dec }}$ and has been previously measured [1].

$$
Y_{\mathrm{dir}}=\frac{R_{\gamma} Y_{\mathrm{inc}}-Y_{\mathrm{dec}}}{R_{\gamma}-1} .
$$

To measure the modification of the fragmentation function of the opposing parton, the awayside yield of the resulting $\gamma_{\text {direct }}-h$ jet function is measured in the head region, $|\Delta \phi-\pi|<\pi / 5$, and plotted as a function of $z_{T}$. For correlations between trigger and associated particles, $z_{T}=$ $\left\langle p_{T, \text { assoc }}\right\rangle /\left\langle p_{T, \text { trig }}\right\rangle$. For $\gamma_{\text {direct }}-h, z_{T}=\left\langle p_{T, h}\right\rangle /\left\langle p_{T, \gamma}\right\rangle \cong\left\langle p_{T, h}\right\rangle /\left\langle p_{T, j e t}\right\rangle$ and is used to approximate $z$. The $z_{T}$ distributions are shown in Figure 1. The upper set of points are for $p+p$ collisions from the 2005 and 2006 data combined and the lower set are for $A u+A u$ collisions from the 2007 data. The 2007 data set is a factor of four larger than the previously shown 2004 data. The improved data set allows for a measurement at higher $p_{T, h}$, extending the results to higher $z_{T}$.

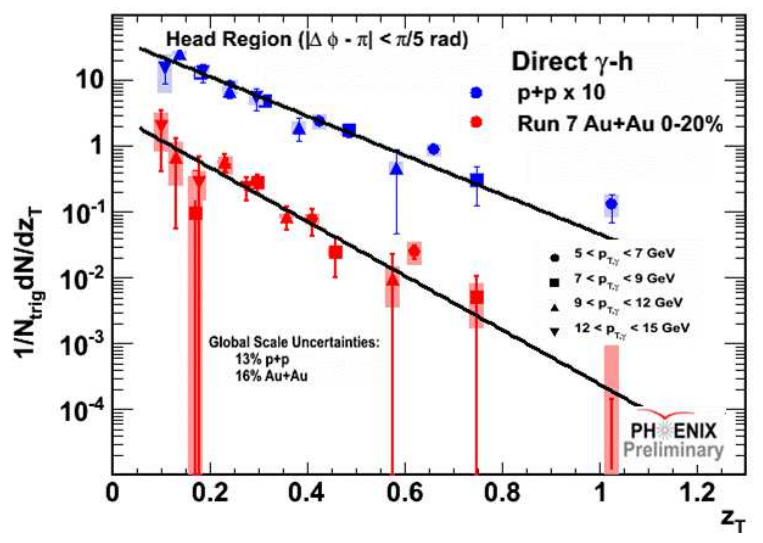

Figure 1: (Color online) The $z_{T}$ distribution for $p+p$ scaled by a factor of 10 (blue) and $A u+A u$ (red).

All $p_{T}$ bins appear to obey approximate $z_{T}$ scaling. Therefore, all points for each collisional system were fit with the function, $\frac{d N}{d z_{T}}=N e^{-b z_{T}}$. For $p+p$ the slope was measured to be $b=$ $6.89 \pm 0.64$. The slope in $A u+A u$ is $b=9.49 \pm 1.37$ which exceeds that in $\mathrm{p}+\mathrm{p}$, as expected for modified fragmentation in the QCD medium due to constant fractional energy loss of the away-side parton. However, the statistical uncertainties in these data limit the significance of the difference to only $1.3 \sigma$. 
To quantify the suppression observed in the $\gamma_{d i r}-h$ channel, the $I_{A A}$ is measured. The $I_{A A}=Y_{A u+A u} / Y_{p+p}$ is the ratio of the conditional yield observed in $A u+A u$ to that in $p+p$. As with $R_{A A}, I_{A A}=1$ would indicate no modification. Figure 2 shows suppression for $A u+A u$ yield since $I_{A A}<1$. This suppression is compared to the suppression measured in $\pi^{0}-h$ in the same figure. With the possible exception of the lowest and highest $z_{T}$ points, the $I_{A A}$ of $\gamma_{d i r}-h$ and $\pi^{0}-h$ are remarkably consistent. This is surprising since $\pi^{0}-h$ should include more gluon jets which are more suppressed. However, $\pi^{0}-h$ suffers a surface bias, which reduces the suppression observed, while $\gamma_{d i r}-h$ does not suffer such bias since the $\gamma_{d i r}$ is not suppressed by the medium.
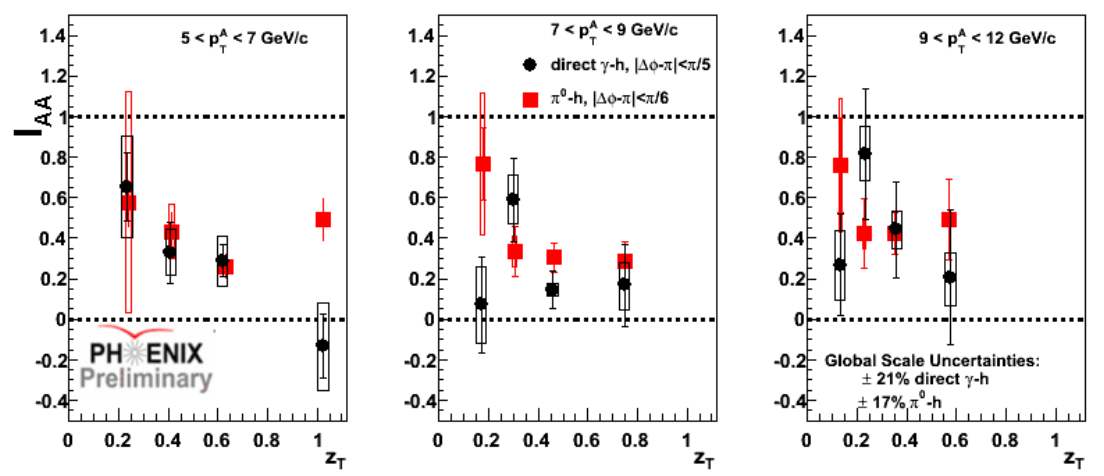

Figure 2: The $I_{A A}$ measured in the head regions for $\gamma_{d i r}-h$ and $\pi^{0}-h$ as a function of $z_{T}$ for three different $p_{T, t r i g}$.

The $I_{A A}$ for $\gamma_{d i r}-h$ is also compared to several of the current theories as shown in Figure 3 . The ZOWW curve [5] shows suppression at high $z_{T}$ and less suppression at low $z_{T}$. This shape results from the geometric dependence of the hard scatterings. High $z_{T}$ is more surface biased since it requires the associated hadron to have higher momentum while low momentum hadrons (low $z_{T}$ pairs) probe deeper into the medium, reducing the observed suppression. Renk's curve [6], however, does not have a strong $z_{T}$ dependence because he allows for fluctuations in his energy loss model, washing out the geometric dependence effects. Finally, the data are compared to MLLA results from [7] in which the energy loss goes into production of low momentum particles. This enhances the low $z_{T}$ region such that at very low $z_{T}$, the $I_{A A}$ is above unity. The current measurements can not fully rule out any of these theories. However, these measurements will benefit from additional statistics from future runs and possibly improvements of the subtraction method.

We also study $\gamma_{d i r}-h$ correlations at small opening angles. The near-side yield of associated particles for $p+p$ and $A u+A u$ are shown in Figure 4 All points are consistent with little to no yield on the near-side; it may be non-zero since fragmentation and possible medium induced photons contribute. The $A u+A u$ data also show no indication of enhancement compared to the $p+p$ points.

In conclusion, suppression has been observed on the away-side of the $\gamma_{d i r}-h$ channel in $A u+A u$ collisions at $200 \mathrm{GeV}$. This suppression is remarkably consistent with the suppression measured in the $\pi^{0}-h$ correlations. The $z_{T}$ distributions shown suggest a steeper slope in $A u+A u$, 


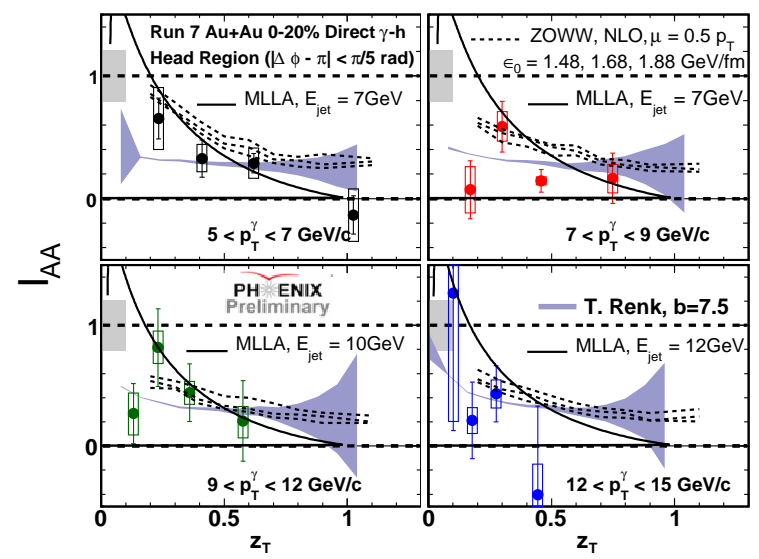

Figure 3: The $I_{A A}$ measured in the head regions for $\gamma_{d i r}-h$ (circles) compared to three different predictions [5, 6, 7].
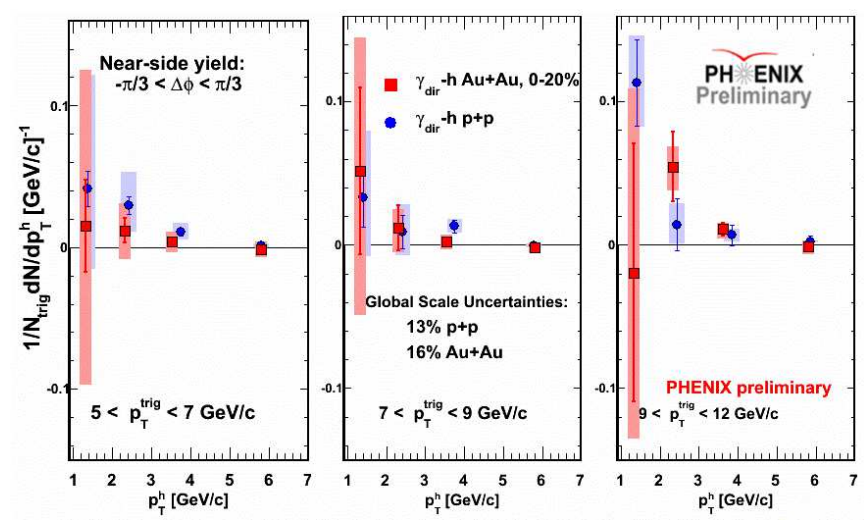

Figure 4: The nearside yield in $p+p$ (circles) and $A u+A u$ (squares) as a function of $p_{T, h}$ for different $p_{T, \gamma}$.

$9.49 \pm 1.37$, compared to the slope, $6.89 \pm 0.64$, measured in $p+p$ collisions. Preliminary measurements of the nearside show no evidence of ehancement in $A u+A u$ compared to $p+p$. With increased statistics and extended kinematic reach, experimental results of $\gamma_{d i r}-h$ are moving toward precision measurements of energy loss.

\section{References}

[1] S.S. Adler et al, Phys.Rev.Lett.94, 232301 (2005)

[2] X.N. Wang, Z. Huang PRC55 (1997) 3047-3061

[3] Justin Frantz, arXiv:0901.1393 v1 [nucl-ex]

[4] Adare et. al., $\operatorname{arXiv:0903.3399}$

[5] Zhang et. al, arXiv0902.4000v1

[6] Renk, arXiv:0904.3806

[7] Borghini and Wiedemann arXiv:hep-ph/0506218 